ECCOMAS

Proceedia

\title{
SEISMIC AMELIORATION OF EXISTING REINFORCED CONCRETE BUILDINGS: STRATEGY TO OPTIMIZE THE AMOUNT OF REINFORCEMENT FOR JOINTS
}

\author{
Vincenzo Bianco $^{1}$, Giorgio Monti ${ }^{1}$, Alessandro Vari $^{2}$ and Gianluigi Palmieri ${ }^{2}$ \\ ${ }^{1}$ Department of Structural Engineering and Geotechnics - Sapienza University of Rome, Italy \\ via Antonio Gramsci n`53 00197 - Rome \\ \{vincenzo.bianco, giorgio.monti\}@uniroma1.it \\ ${ }^{2}$ Research and Development Department - Edil CAM Sistemi s.r.1. \\ via dei Genieri n³9 00143 - Rome \\ \{a.vari,g.palmieri\}@edilcamsistemi.com
}

Keywords: Reinforced Concrete, Existing Buildings, Seismic Amelioration, Design, BeamColumn Joints.

\begin{abstract}
Most of the existing Reinforced Concrete $(R C)$ buildings in Italy were built according to obsolete regulations that were not enough aware of issues related to seismic design so that they need to be upgraded by pursuing either amelioration or full seismic rehabilitation. In doing that, the first step is to figure out what is, based on the results of the initial analysis of the structure in its ante-operam version, the best overall dissipative mechanism that could be obtained by a number of suitable and economically convenient local interventions. The choice of the overall dissipative mechanism strongly affects the amount of reinforcement to be adopted for the beam-column joints. For new buildings, the current adopted capacity design philosophy pursues an overall beam-sway mechanism in which plastic hinges first form in beams and at last at the base of the columns. On the contrary, for existing ones, often very irregular and gravity-load-dominated, pursuing such overall mechanism may result either uneconomic or even extremely difficult to implement due to the amount of reinforcement to be inserted in the joints. In such cases, an overall dissipative mechanism allowing, at some extent, columns flexural plasticization should be accepted and clearly identified in advance. Anyway, such approach needs to be addressed properly in order to avoid the formation of column-sways at one story only that would result very dangerous due to the excessive demand of plastic rotations on the resulting hinges. This paper presents two simple models that may help the designer in dealing with the operations above. The former is a model that allows to understand if, given the existing RC building case-study, either the beam-sway or a hybrid beam-column-sway mechanism should be conveniently pursued during the design of the retrofitting intervention. The latter is a model that allows to design a hybrid beam-column-sway overall mechanism involving a suitable number of stories such as to guarantee a uniform and reasonable demand of plastic rotations in the involved columns.
\end{abstract}




\section{INTRODUCTION}

A large amount of the built heritage in most of the European earthquake-prone countries is composed of existing Reinforced Concrete (RC) buildings around a century old. These buildings were certainly built in compliance with very obsolete Building Codes (e.g. [1]) showing no or little awareness of the seismic-behavior-related issues so that they are certainly inadequate to sustain the design earthquake specified by current regulations (e.g. [2,4]). Such buildings require either amelioration or full-rehabilitation interventions. The former means making the building capable to sustain, for a given Ultimate Limit State (ULS), a larger percentage (less than $100 \%$ ) of the Code-specified earthquake while the latter means intervening to such an extent to make the building capable to withstand one hundred percent of the seismic action.

Following the recent seismic events that have struck the Italian territory, i.e. the city of L'Aquila (2009), the region of Emilia Romagna (2012) and the city of Amatrice (2016), professional engineers have undertaken many of such retrofitting intervention designs. Independently of the aim of the intervention, either amelioration or full rehabilitation, there has been a widespread tendency to use innovative techniques for the local strengthening of deficient RC members, either beams, columns, or joints. Among these techniques in particular, either 1) the use of Fiber Reinforced Polymer (FRP) wrappings, or 2) the so-called CAM ${ }^{\circledR}$, which is the acronym of the Italian name Cucitura Attiva dei Manufatti (literally: Artefacts Active Sewing), have been attracting the attention of the technical community and also applied, in many casestudies (Fig. 1). According to the latter, existing RC elements are wrapped by high strength steel strips that are slightly pretensioned in order to confine the retrofitted element thus providing a readily available source of extra-strength that, differently from the FRPs, does not need the element deformation in order to be activated. The $\mathrm{CAM}^{\circledR}$ has also the advantage to allow the RC element be literally sewed whatever the geometrical boundary conditions characterizing the specific case study might be. The FRPs on the contrary, even if not active, offer the advantage to be lighter and easier to apply. Anyway, those techniques are still more expensive with respect to the more traditional ones contemplating the jacketing of existing RC elements by either 1) plain steel cold formed elements joined in situ by fillet welds or 2) reinforced concrete coatings. For this reason, since very conservative interventions may result excessively expensive thus discouraging both the application and the further spread of those innovative techniques, there is a growing attention towards the way to optimize the amount of the reinforcement.

The intense post-earthquake retrofitting design activity that has taken place in Italy has shown that the necessity to optimize the amount of reinforcement is particularly important for the case of RC joints. The Italian Building Code offers two alternative strategies to evaluate the amount of stirrups to strengthen the joint panel that are: 1) the formulation presented in the main cogent document [2], which are transposed from the European Codes [3,4], and 2) the formulation reported in the explanatory (not cogent) document [5]. The former is a formulation that seems to be derived from the pioneering works on the capacity design philosophy [6] and that is essentially based on the resisting mechanisms that characterize the joint behavior at its ultimate state when, due to the presence of thoroughly smeared cracks, it is far from being constituted of an homogenous and isotropic continuum material. While the latter, implicitly proposed for the case of existing buildings, is based on the application of the Mohr circle to a joint assumed composed of an homogeneous and isotropic elastic material. The former formulation seems to be well contextualized in the ambit of the performance-based design philosophy, which is already adopted by the Euro Codes, and is based on the conception of an overall dis- 
sipative mechanism according to which the new building is expected to behave during an earthquake attack. On the contrary, the latter one seems to be an inheritance of the former prescriptive design philosophy according to which, in the case of existing buildings, we can just strengthen the joints, regardless of the overall dissipative mechanism induced. Such approach indeed is justified by the difficulty to intervene on existing buildings that can be very irregular, either in plan or in elevation, and that, due to the gravity-loads-based original design, are often characterized by the presence of strong beams and weak columns. On the contrary, for the conception of new buildings, the formation of an overall dissipative mechanism that envisages the formation of plastic hinges in the beams extremities first and then at the lowermost extremity of the columns, is favored by the adoption of a weak-beam-strong column regular conception. In the case of existing buildings, the design of interventions capable to induce a proper overall dissipative mechanism is relatively more cumbersome.

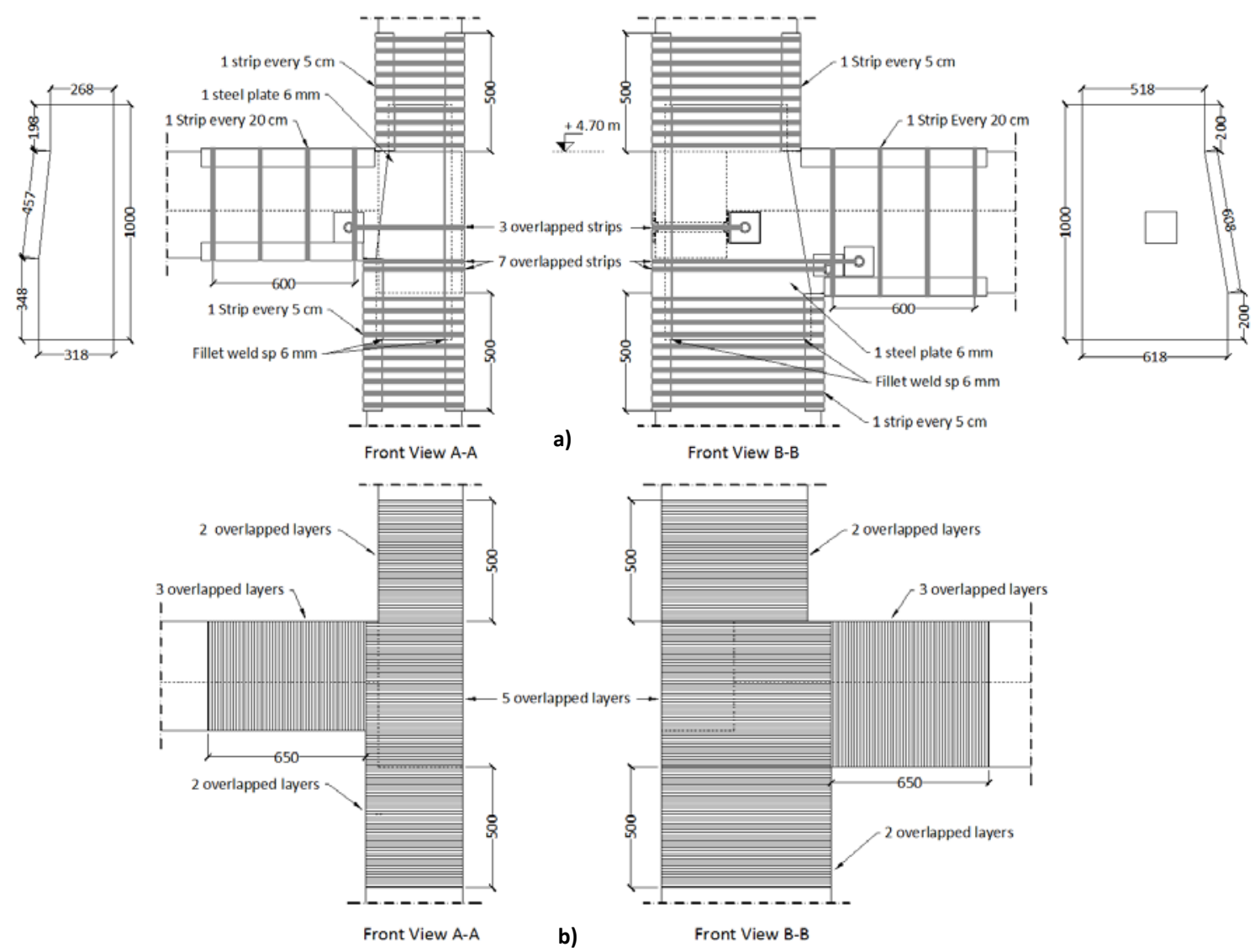

Figure 1 Most common innovative techniques recently adopted in Italy to strengthen RC joints: a) $\mathrm{CAM}^{\circledR}$ wrapping by high strength steel strips and b) FRP layers.

In this scenario, even though design is carried out at the Life Safety ULS, in which the use of the first formulation would be more appropriate, practitioners have diffusely implemented the second one that has brought to more reasonable amounts of reinforcements, even though in a complete unawareness of the overall dissipative mechanism thus induced.

With the aim to contribute to a more rational design of the seismic retrofitting interventions on existing RC buildings, the present work proposes a strategy to optimize the amount of reinforcement for joints by taking also into consideration the overall dissipative mechanism to be pursued by means of local interventions. 


\section{OVERALL DISSIPATIVE MECHANISM}

\subsection{New RC buildings}

When it comes to new RC buildings, the widely accepted tendency is to design ductile Moment Resisting Framed (MRF) structures, by means of the so-called Capacity Design, which was early introduced by Paulay and Priestly [6]. The objective is to design MRF structures that are capable to withstand acceptable excursions in the plastic range, during the earthquake-induced oscillations, thus dissipating the input energy.

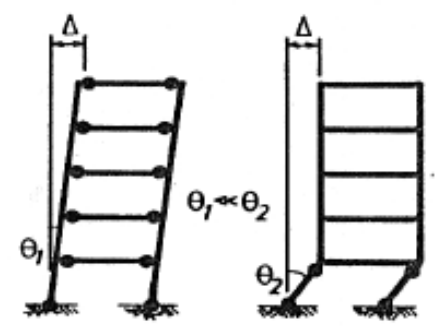

Figure 2 Energy-dissipating mechanisms: a) mechanism with smeared formation of plastic hinges, in all beams and at the base of the columns, b) soft story mechanism.

The overall energy dissipating mechanism is selected in advance. The most favorable mechanism is the one in which flexural plastic hinges form at the extremities of all the beams first, and then at the lowermost extremity of all the columns. This mechanism (Fig. 2a) is the most suitable one since it guarantees a uniform distribution of plastic hinges throughout the development of the building, both in plan and elevation, thus fulfilling the overall displacement ductility requiring the smallest inelastic rotational demands in the plastic hinges. On the contrary, the so-called soft-story mechanisms (Fig. 2b), in which plastic hinges form at both the extremities of all the columns of a story, should be avoided since they imply that, for the same overall displacement ductility requirement, the local rotational demand in the plastic hinges is much larger. Such plastic hinges rotational demands may be too large so that, even with good detailing of the affected regions, would be difficult to accommodate. Another aspect that would further complicate the management of a soft-story mechanism is the $P-\Delta$ effect associated with the corresponding large horizontal displacement. Contextually to the selection of the overall dissipative mechanism, the location of the plastic hinges has to be determined with a relatively high degree of precision. Parts of the structure that are intended to remain elastic are designed so that under maximum feasible actions corresponding to the development of overstrength in the plastic hinges, no inelastic deformations should occur in those regions. Therefore, it is immaterial whether the failure of regions, intended to remain elastic, would be ductile or brittle. The actions originating from plastic hinges are those associated with the overstrength of these regions. The required strength of all other regions is then in excess of the strength demand corresponding to the overstrength of relevant plastic hinges. The care in the detailing of the plastic hinge regions is larger than that dedicated to the regions that are to remain elastic.

To guarantee the dissipative mechanism above, the formation of plastic hinges, in all the beams and columns, should occur almost simultaneously, which means for the same value of the overall displacement ductility demand. In this scenario, what are the operational instruments that designers have to induce such overall behavior? At this early stage of design, they can 1) manipulate the building geometry, and 2) redistribute the design actions. The former means trying to have a very regular structure where the concept of regularity also implies, beyond the even distribution of mass and stiffness [2-5], both in plan and elevation, that beams and columns be of practically the same length. This is necessary in order to have almost the same value of 
rotational demand in all the beams plastic hinges, for a given value of the overall displacement demand. The redistribution means that, relying on the elasto-plastic behavior of the plastic hinge zones, bending moments can be transferred, on a given story, from one joint to another, thus reducing the capacity required to a beam extremity that will yield earlier and plastically rotate thus transferring the bending moment in another point. The moment redistribution is also useful to achieve a more efficient structural design of the steel reinforcement [6].

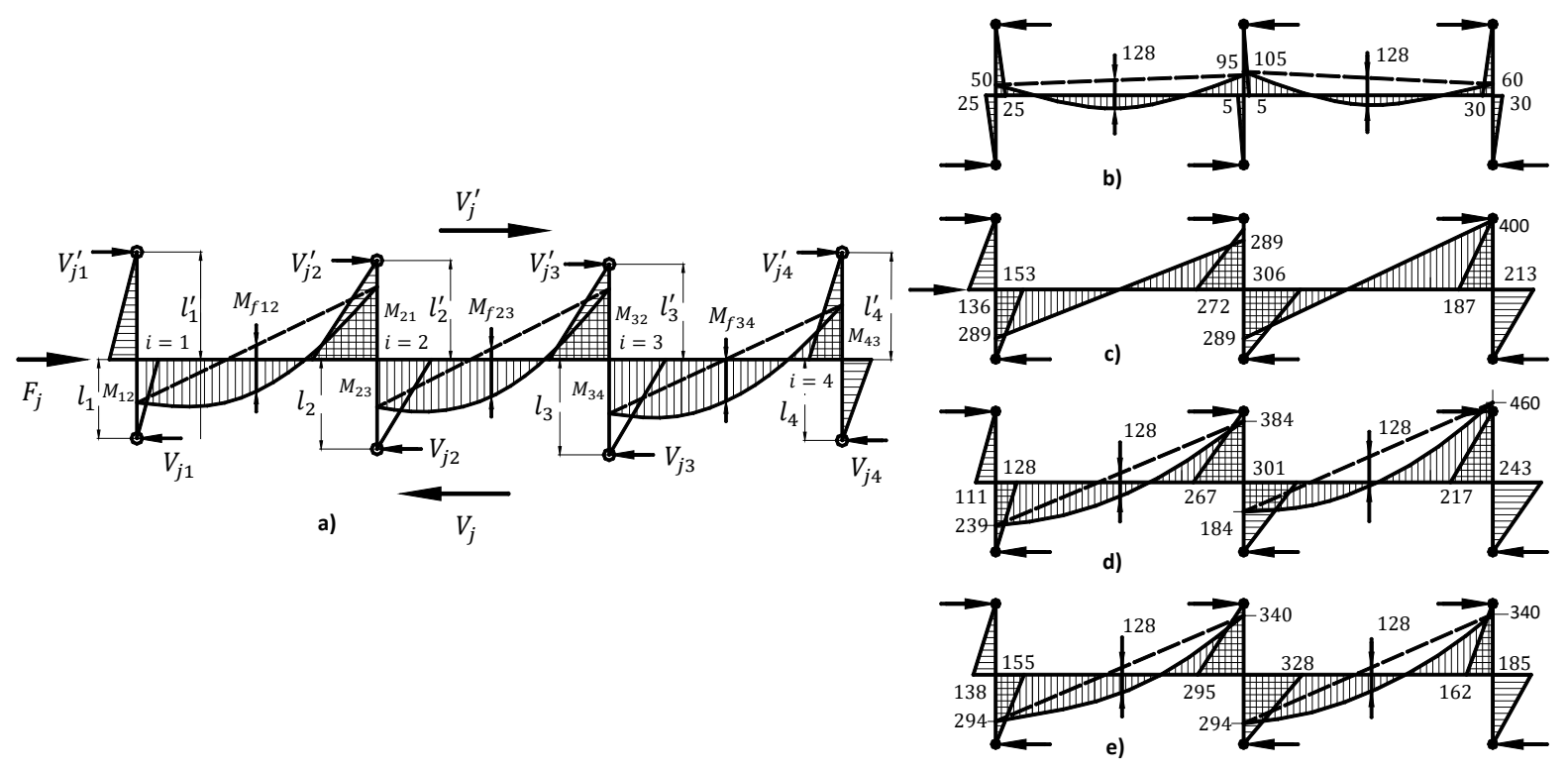

Figure 3 Features of moments redistribution: a) equilibrium of a subframe, b) gravity moments, c) earthquake moments, d) combined gravity and earthquake moments, e) redistributed gravity and earthquake moments.

The essential requirement of the moment redistribution process is that equilibrium under the applied seismic forces and gravity loads must be maintained. Fig. 3a represents a typical subframe of a multistory frame isolated by cutting columns above and beneath the beams at column contraflexure points. The moment pattern shown resulted from an elastic analysis for the simultaneous action of gravity loads and earthquake loads, duly factored. The total shear forces transmitted by the columns below and above the floor are $V_{j}$ and $V_{j}^{\prime}$, respectively, for the rightward direction of the earthquake forces considered. In considering equilibrium criteria, the simplifying assumption is made that the distance between the two column points of contraflexure, above and below the beam centerline, is the same for each column of the frame, and this distance does not change while beam moments are redistributed. This means that $l_{i}^{\prime}+l_{i}=l_{c}$ is a constant length. During moment redistribution, the moment applied to the ends of the beams may be changed, but the total moment input to the columns must remain unchanged, and equal to the sum of the moments input by the column to the beam at joint $i$, that is:

$$
\sum_{i}\left(M_{i, i-1}+M_{i, i+1}\right)=\sum_{i} M_{b i}=\sum_{i} M_{c i}
$$

where: $M_{b i}$ is the bending moment introduced to a column by the beams that are joined to the column at node point $i$, and $M_{c i}$ is the moment input to the continuous beam at joint $i$.

There are two characteristic situations for moment redistribution along continuous beams: a) the first involves beam moments redistribution across a joint, and b) the second type of moment redistribution in the beam involves redistribution of actions between columns. The former implies that, if a beam moment at a joint is changed, the other at the same joint, must change 
accordingly but, since the total beam moment input to the joint remains unaltered, the moments and shear forces for the relevant column remain the same as before. The latter means that, when a beam moment change (e.g. a reduction) at a given joint $i$ is compensated by a beam moment change in other joints (an increase), even though Eq. (1) is satisfied, since the moment input to these latter columns has changed, the shear forces in these latter will also change. Thus, redistribution of both moments and shear forces between columns will take place.

In an attempt to achieve a desirable solution, the designer manipulates end moments of beams. Gravity load equilibrium will be maintained provided that the part of the bending moment that originates from gravity load only, and is applicable to a simply supported beam (i.e. the moment superimposed on a straight baseline which extends between the beam end moments), is not changed. A change of beam end moments in any span will change the beam reactions and hence the forces introduced to individual columns below the beam under consideration.

An example of moment redistribution for a regular earthquake dominated frame is shown in Fig. 3b-e. The moment pattern (Fig. 3d) due to the superimposition of the patterns due to gravity loads (Fig. 3b) and to the earthquake equivalent lateral force (Fig. 3c) is redistributed (Fig. 3e) in order to have almost equal values of positive and negative bending moments, thus yielding a more uniformly distributed steel reinforcement along the beam length.

The application of the Capacity Design, intended to induce the formation of the desired overall dissipative mechanism, encompasses the following steps, to be undertaken in sequence: 1) beam flexural design, 2) beam shear design, 3) column flexural design, 4) column shear design, 5) beam-column joint design. Beam flexural design is carried out in such a way that, at the selected plastic hinge locations, flexural strength is as close as possible to the moment requirement resulting from the redistribution process while elsewhere, flexural strength is selected so as not to allow plastic hinges to form. Shear strength at all sections of the beam is designed to be higher than the shear corresponding to the flexural overstrength, due to the steel strain hardening, at the beam plastic hinges. Consideration of joint moment equilibrium and possible higher mode amplification are used to determine the maximum feasible column moment corresponding to beam flexural overstrength. Ideal column moment capacity is matched to these required strengths to ensure that the weak beam-strong column hierarchy is achieved. The determination of the necessary amount of transverse reinforcement is based on the more stringent of the requirements for shear strength, which are: confinement of compressed concrete, stability of compression reinforcement, and lapped bar splices. An estimate of the maximum feasible shear force in the column is made based on equilibrium considerations at beam flexural overstrength. Because beam-column joints are poor sources of energy dissipation, inelastic deformations due to joint shear forces or bond deterioration must be minimized. The ideal strength of joints is matched to the input from adjacent beams when these develop flexural overstrength at the critical sections of plastic hinges.

How can the Capacity Design philosophy described hereinabove be interpreted in energetic terms [7-9]? Referring to an elasto-plastic Single Degree Of Freedom System (SDOF), subject to a seismic action, the energy balance can be written as follows [9]:

$$
E_{I}(t)=E_{S}(t)+E_{K}(t)+E_{D}(t)+E_{H}(t)
$$

where: at a generic time instant $t, E_{S}(t)$ and $E_{K}(t)$ are the instantaneous values of a) strain energy stored by the structure and $b$ ) the kinetic energy of the moving mass, respectively; $E_{I}(t)$, $E_{D}(t)$, and $E_{H}(t)$ are the value of energy, cumulated since the time instant $t_{0}$, and respectively $c)$ input in the structure, $d$ ) dissipated by viscous damping and e) dissipated by hysteresis. The hysteretic and viscous components of the energy, are increasing functions of time since they represent forms of energy that cannot be recovered while, on the contrary, both kinetic an strain 
energy can be recovered. After the end of the earthquake, at time $t_{f}$, the structure undergoes free oscillations with decreasing amplitude with time, which vanish at the time instant $t_{q}>t_{f}$, when all kinetic and elastic deformation energy has been dissipated. The cumulative equation of the energy balance in the instant in which stillness has been restored, can be written as follows:

$$
E_{I}\left(t_{q}\right)=E_{D}\left(t_{q}\right)+E_{H}\left(t_{q}\right)
$$

that shows the overall input energy has been dissipated by both viscous and hysteretic energy. In particular, if the structure has remained in the elastic range throughout the earthquake duration, it is $E_{H}\left(t_{q}\right)=0$ and all the input energy has been dissipated by viscous damping $E_{I}\left(t_{q}\right)=$ $E_{D}\left(t_{q}\right)$. On the contrary, if the structure has undergone excursions in the plastic range, it is $E_{D}\left(t_{q}\right) \ll E_{I}\left(t_{q}\right)$ and the structure has dissipated most of the input energy by hysteresis, which means $E_{H}\left(t_{q}\right) \cong E_{I}\left(t_{q}\right)$.

Thus, since $E_{S}(t)$ and $E_{K}(t)$ are instantaneous values of strain and kinetic energy, and since there is an instantaneous transfer of energy from the former to the latter form of energy, their instantaneous sum is constant. In this way, the objective to induce the formation of the desired dissipative mechanism in the structure by flexural yielding in predefined points, is equivalent to imposing that the potential elastic energy associated to the desired dissipative mechanism be the minimum of all possible mechanisms. Moreover, such potential elastic energy has to be slightly smaller than the kinetic energy corresponding to the Damage Limit State (DLS), that is:

$$
E_{\text {Sey }}=\min \left(E_{S e y, j}\right) \leq E_{K, D L S}=\frac{1}{2} \cdot M \cdot S_{V, D L S}^{2}
$$

where: $E_{S e y, j}$ is the elastic strain energy at yielding for the generic $j$-th dissipative mechanism, $M$ is the mass of the structure, and $S_{V, D L S}$ is the value of the velocity spectrum.

\subsection{Existing RC buildings}

When it comes to existing RC buildings, since they were built in compliance with obsolete Building Codes [1], unaware of seismic behavior, they are often neither regular nor designed to dissipate energy according to a well-defined dissipative mechanism. Moreover, since they were most probably built to resist gravity loads only, they typically present a strong beam-weak column typology. In cases in which there are beams longer than the others, they are most probably gravity-load-dominated which means that, at the beam-column joints, the negative bending moment is much larger than the seismic positive bending moment and their combination yields a negative moment. In this case, after the formation of the first (negative) hinge at one of the beam extremities, the formation of the second one would require a large increase of lateral force or displacement. Even though a skillful application of the either moment redistribution or relocation of plastic hinges away from column faces to zones where both gravity loads and lateral forces generate positive moments may help, in many situations the most meticulous allocation of beam hinge strength will not offset the excess potential lateral force resistance.

In such cases, insisting on pursuing the dissipative mechanism described in the previous section may result extremely difficult if not impossible. The design of the retrofitting intervention would be particularly difficult for joints. Thus, the possibility to intervene inducing other dissipative mechanisms would be preferable. 


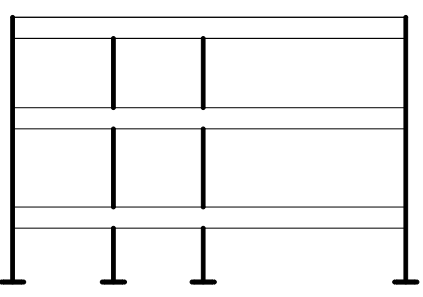

a)

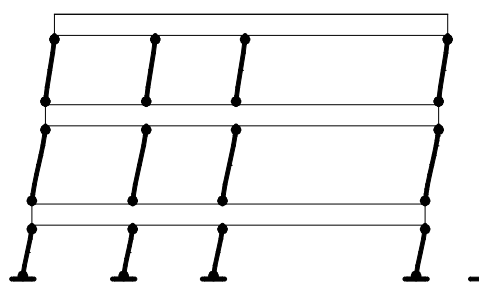

b)

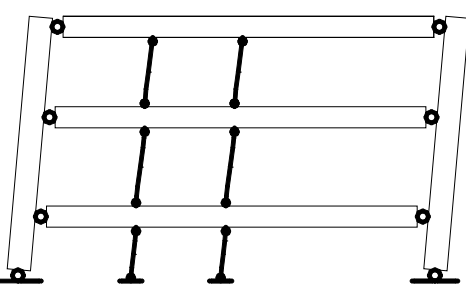

c)

Figure 4 Existing strong-beam-weak-column RC buildings: a) typical example frame, b) shear-type (columnsway) dissipative mechanism, and c) hybrid column and beam sway dissipative mechanism.

Alternative solutions may be the following:

1) A shear-type mechanism (Fig. 4b), in which hinges are allowed to form in the columns extremities even though the right expedient has to be found in order to both a) force the formation of plastic hinges at the extremities of the columns of several stories, if not all, and b) ensure that, even after the formation of plastic hinges, the post-elastic oscillations do not concentrate on one story only. In fact, in this latter case, a soft story mechanism would take place.

2) A hybrid column and beam sway mechanism (Fig. 4c) in which at least a partial beam sway mechanism should develop to ensure that no column sway mechanism (soft story) can form. This can be achieved if plastic hinges are made to develop in the outer spans of beams close to exterior columns, which in turn may need to be strengthened in order to have adequate flexural strength to absorb without yielding the moment input from these outer beam spans. Column hinges above level one will thus not develop in the two outer columns of the frame. At the inner beam-column joints, column hinges below and above each floor will need to form to complete the frame mechanism. Since such mechanism has to rely on the flexural ductility capacity of the columns, which may not be desirable, the overall ductility demand may be restricted by increasing the lateral resistance of the frame to lateral forces.

Each of these alternative overall dissipative mechanisms will yield the beneficial effect to reduce the horizontal strengthening in most of the joints since the beam cross section at the column face does not yield. This aspect will be clarified in next sections.

\section{CHOICE OF THE OVERALL DISSIPATIVE MECHANISM}

In this paragraph a simple model is proposed that may help the designer figure out if, for the given case-study, it is convenient to orient the retrofitting intervention towards the reproduction of a beam-sway mechanism or instead it is more rational to purse one of the alternative overall dissipative mechanisms. A typical existing RC building characterized by beams and columns of different length and by the Strong-Beam-Weak-Column features is represented in Fig. 5.

The idea is to evaluate if, given the geometrical and mechanical characteristics of the various elements, once shear failures have been excluded [10,11], plastic hinge formation occurs in the columns earlier than in the beams, which means if:

$$
u_{c, y c} \ll u_{c, y b}
$$

where: $u_{c, y c}$ and $u_{c, y b}$ are the values of displacement of the control point $u_{c}$ corresponding to the yielding of columns (the first one to yield, actually) and beams, respectively. If that is the case, trying to induce a classical beam sway mechanism is worthless. 


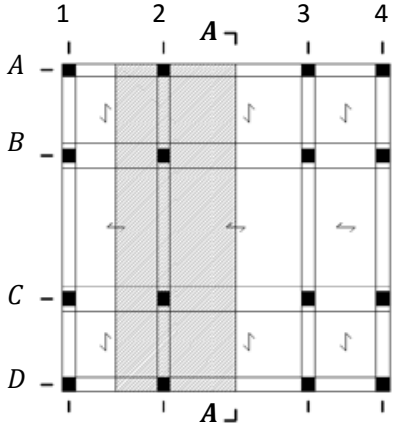

a)

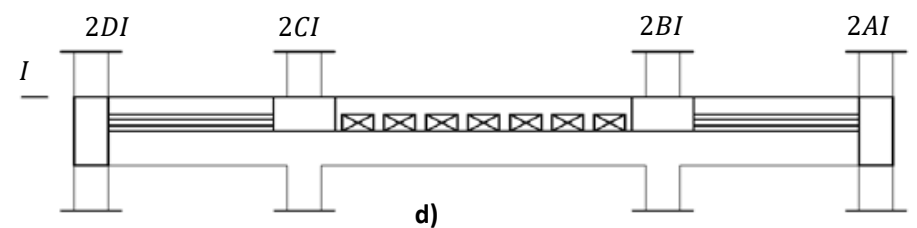

d)

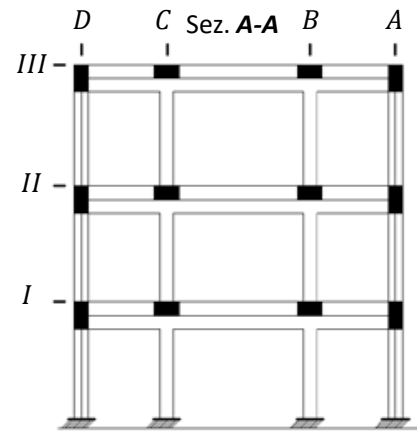

b)

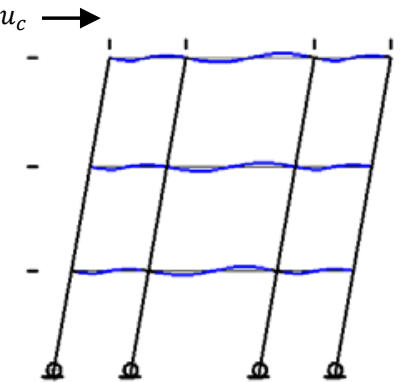

c)

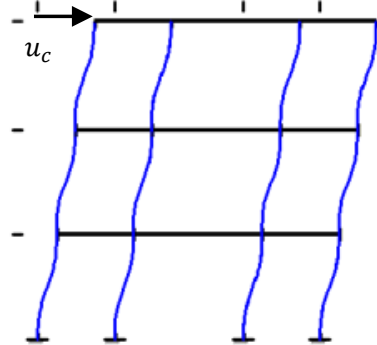

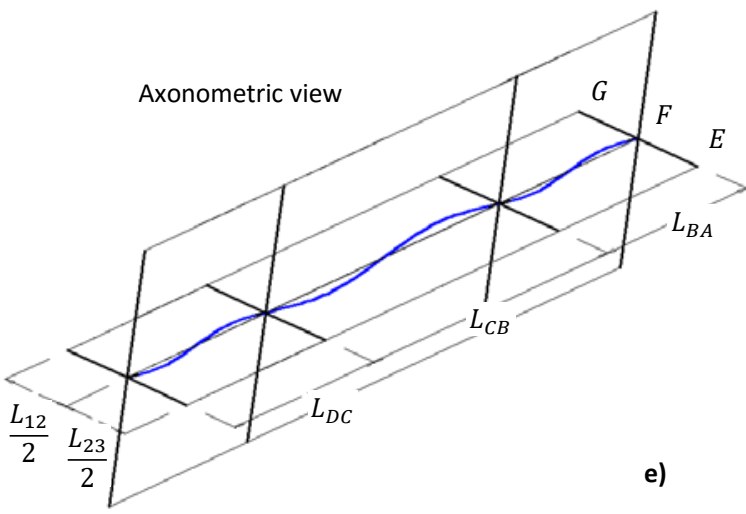

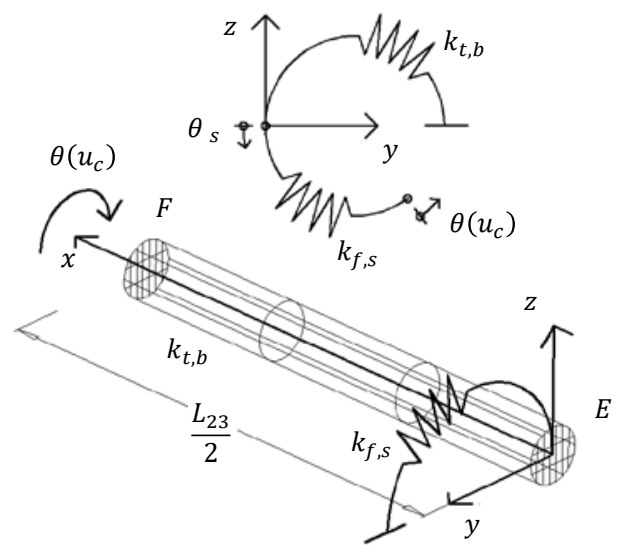

Figure 5 Typical existing Strong-Beam-Weak-Column RC building: a) plan and elevation, b) simplified frame model to study beams, c) simplified model to study columns, d) details of the RC slab (topping) along with the supporting beams, and e) details of the model for beams taking into account the torsional retainer offered by beams orthogonal to the earthquake direction.

The values of $u_{c, y c}$ and $u_{c, y b}$ can be calculated by simplified plane frame and conservative models $[10,11]$ that contemplate the concentration of deformations on columns or beams only, respectively (Fig. 5bc). As general assumptions, it is assumed that: (1) all frame elements are inextensible, and (2) floors are infinitely rigid in their plane. In this way: (1) all joints just move horizontally, and (2) both their horizontal and vertical mutual distances remain unchanged. In particular for beams (Fig. 5b), since the model herein presented has to be as simple as possible, the conservative model is adopted in which the whole seismic-induced deformation is concentrated on beams. This means adopting a model in which columns are hinged at the base and just rigidly rotate around their lowermost extremity during the imposition of increasing horizontal displacements. For the generic horizontal beam, due to the assumption of inextensibility of columns, vertical displacements in their extremities are null, while end rotations are assumed to be equal to each other. When the earthquake strikes, it imposes, through the resulting inertia forces, a rotation at the beam extremity, and the bending moment due to such rotation is given by [12]: 


$$
M_{S d}=\frac{8 \cdot E_{b} \cdot I_{b}}{L_{b}} \cdot \theta=\frac{6 \cdot E_{b} \cdot I_{b}}{L_{b} \cdot H_{t o t}} \cdot u_{c}
$$

where: $E_{b}$ is the beam cross section Young's modulus, $I_{b}$ the beam cross sectional inertia, $L_{b}$ the beam length, and $H_{\text {tot }}$ the total height of the building.

If there are also edge beams, due to the flexural stiffness of the deck framing on them, these constitute a torsional restraint that ends up limiting the rotation of the beam extremity. So that, when such restraints cannot be neglected (Fig. 5), the counter-moment applied by the assembly of edge beam and deck has to be subtracted from Eq. (6). Such torsional restraint-induced bending moment can be evaluated by considering the very simple model (Fig. 5e) composed by two torsional springs, arranged in series to each other, and one with the torsional stiffness of the half edge beam $k_{t, b}$ and the other with the flexural stiffness (in the direction parallel to the earthquake) of the slab $k_{f, b}$. This latter can be, in first instance, concentrated in the internal extremity of half beam length (point $\mathrm{E}$ in Fig. 5e), and is given by:

$$
k_{f, s}=\frac{4 \cdot E_{s} \cdot I_{s}}{L_{s}}
$$

where: $L_{S}$ is the length of the slab framing into the edge beam, and $E_{S}, I_{S}$ are the slab cross section Young's Modulus and sectional inertia, respectively.

While the edge beam torsional stiffness is given by:

$$
k_{t, b}=\frac{I_{t} \cdot G_{t}}{L_{t}}
$$

where: $L_{t}$ is the edge beam length, and $I_{t}, G_{t}$ are the beam cross section inertia and transversal stiffness modulus, respectively.

Due to the combination in series of the two springs above, the kinematic compatibility yields that the overall earthquake-imposed end rotation is equal to the sum of a) the torsional rotation undergone by the edge beam $\theta_{b}$ and $b$ ) the flexural end rotation undergone by the portion of slab insisting on the edge beam $\theta_{s}$, which means:

$$
\theta\left(u_{c}\right)=\theta_{b}+\theta_{s}
$$

Moreover, for the equilibrium to be fulfilled, the torsional moment $M_{t, b}$ in the edge beam has to be equal to the bending moment in the slab $M_{f, s}$, which means:

$$
M_{t, b}=M_{f, s}
$$

Thus, by combining Eq. (9) and Eq. (10), we obtain:

$$
\theta\left(u_{c}\right)=\theta_{b}+\theta_{s}=\frac{M_{t, b}}{k_{t, b}}+\frac{M_{f, s}}{k_{f, s}}
$$

from which the value of the counter-moment can be evaluated as follows:

$$
M_{t, b}=M_{f, s}=\theta\left(u_{c}\right) \cdot\left(k_{f, s} \cdot k_{t, b}\right) /\left(k_{f, s}+k_{t, b}\right)=\frac{u_{c} \cdot\left(k_{f, s} \cdot k_{t, b}\right)}{H_{t o t} \cdot\left(k_{f, s}+k_{t, b}\right)}
$$

In conclusion, the end bending moment will be given, combining Eq. (6) and Eq. (12), by the following expression: 


$$
M_{S d}=\left[\frac{6 \cdot E_{b} \cdot I_{b}}{L_{b} \cdot H_{t o t}}-\frac{\left(k_{f, s}+k_{t, b}\right)}{H_{t o t} \cdot\left(k_{f, s} \cdot k_{t, b}\right)}\right] \cdot u_{c}
$$

Introducing the beam yield strength $M_{b R y d}$ in Eq. (13) and making it explicit with respect to the control point displacement, the $u_{c, y b}$ value can be obtained.

For the case of the column, given the column yield strength $M_{c R y d}$, the corresponding value of the control point displacement can be obtained by:

$$
u_{c, y c}=u_{c}\left(M_{c R y d}\right)=\frac{L_{c}^{2} \cdot M_{c R y d}}{6 \cdot E_{c} \cdot I_{c}} \cdot \frac{H_{t o t}}{H_{i}}
$$

where: $L_{c}$ is the length of the column, $E_{c}, I_{c}$ are the column cross section Young's Modulus and sectional inertia, respectively, and $H_{i}$ is the $i$-th story height.

\section{CAPACITY MODEL FOR THE JOINT}

Joints of RC buildings can be typically classified according to either one of two criteria: 1) one based on the geometry of the boundary conditions, or 2) one based on the possibility that inelastic deformations occurred in the beam longitudinal bars may or not spread within the joint core. Based on the former criterion, joints can be either 1) exterior, in case one beam only frames into the relevant column, or 2) interior, in case two beams frame into the relevant column. According to the latter criterion, a joint can be either elastic or inelastic if plastic hinges are allowed to form in the beam cross sections far from the column or adjacent to the column, respectively.

\subsection{Original model}

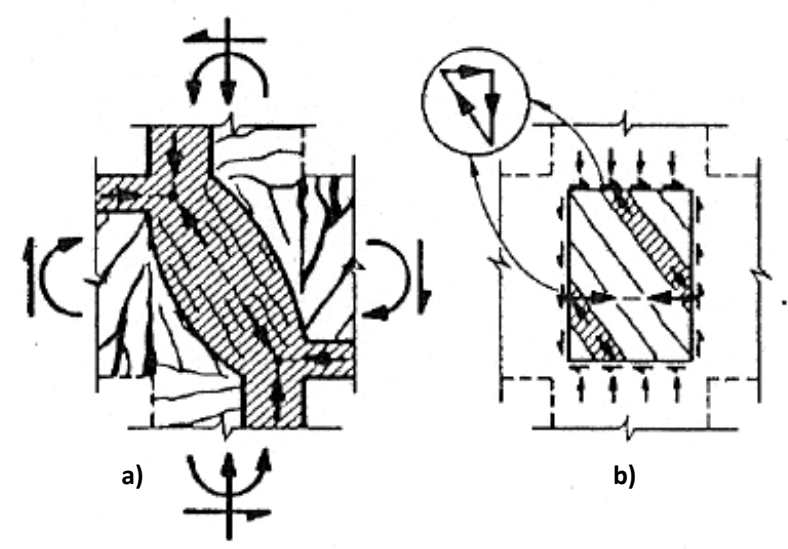

Figure 6 Mechanisms of shear transfer at an interior joint: a) concrete strut and b) diagonal compression field.

In general, two resisting mechanisms can be singled out in a joint, which are (Fig. 6): 1) concrete diagonal compression strut and 2) diagonal compression field. The latter is based on the assumption that concrete joint core is thoroughly cracked so that no (diagonal) tensile stress can be transmitted by the concrete. When beams with very small amounts of flexural reinforcement are used, or when column sections relative to beam sizes are large, joint shear stresses may be rather small and no or very few diagonal cracks may develop. As the concrete core in such cases will resist shear by means of diagonal tensile stresses, the truss mechanism in Fig. 6 will be hardly mobilized. The strut mechanism does not rely on steel contributions, but the truss 
mechanism may require considerable amounts of reinforcement, particularly in the horizontal direction.

In the two limit situations of a) absence of axial compression (even axial tension) or b) large value of axial compression, the compression strut inclination will be less and more steep, respectively. In the latter case, due to the beneficial effect of normal stresses on bond strength, the relative importance of the transfer mechanism of the diagonal compression field will be larger.

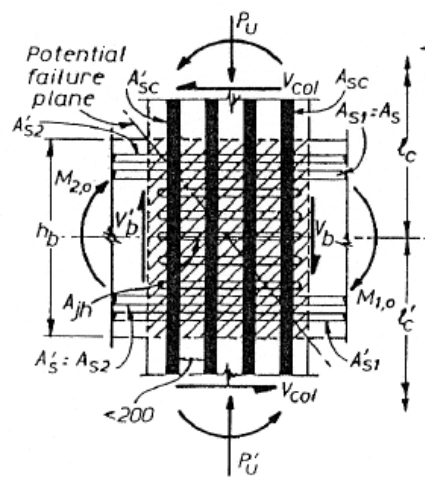

a)

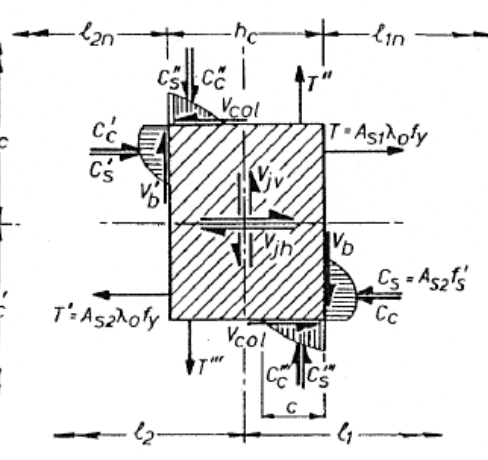

b)

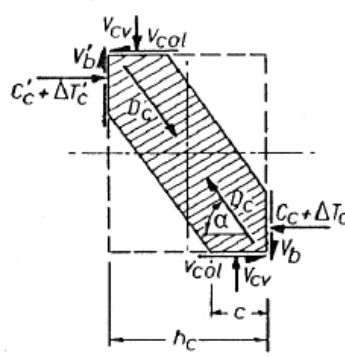

c)

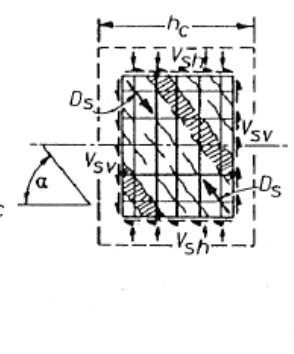

d)

Figure 7 External actions and internal stress resultants at an interior joint.

With reference to the interior joint (Fig. 7), horizontal shear force will be given by:

$$
V_{j S h}=T_{s}+C_{c}^{\prime}+C_{s}^{\prime}-V_{c o l}
$$

in which: $T_{s}$ is the internal horizontal tension introduced in the joint by the beam upper longitudinal reinforcement, $C_{c}^{\prime}$ is the compression force in the concrete, $C_{s}^{\prime}$ is the axial compression in the beam upper longitudinal reinforcement (on the left-hand side of the figure), and $V_{c o l}$ is the average of column shears above and below the joint. Even though the sum of terms $T_{s}+C_{s}^{\prime}$ is transferred to the concrete of the joint core by means of bond stresses, a fraction $\Delta T_{c}^{\prime}$ will be transmitted to the diagonal strut in correspondence of the flexural compression zone of the column above (stress-block). Thus the value of the horizontal shear force transmitted to the diagonal strut mechanism, is given by:

$$
V_{c h}=C_{c}^{\prime}+\Delta T_{c}^{\prime}-V_{c o l}
$$

while the fraction carried by the truss mechanism is:

$$
V_{s h}=T_{s}+C_{s}^{\prime}-\Delta T_{c}^{\prime}
$$

After making reasonable assumptions about the likely distribution of tangential bond stresses inside the joint core and along the beam upper longitudinal reinforcement, Paulay and Priestley [6] suggested the following expression:

$$
V_{c h}=\left(1.55 \cdot \frac{c}{h_{c}}+\beta-0.55\right) \cdot T_{s}-V_{c o l}
$$

in which: $\beta=A_{s}^{\prime} / A_{s}$ is the ratio of the beam compressed longitudinal reinforcement to the tensile steel cross section, $c$ is the depth of the flexural compression zone of the elastic column above, which can be approximated by: 


$$
c=\left(0.25+0.85 \cdot \frac{P_{u}}{f_{c}^{\prime} \cdot A_{g}}\right) \cdot h_{c}
$$

where: $P_{u}$ is the minimum compression force acting on the column $P_{u}=0.25 \cdot f_{c}^{\prime} \cdot A_{g}, f_{c}^{\prime}$ is the concrete cylindrical compressive strength, $A_{g}$ the gross cross section area of the column. From the expressions above, it yields:

$$
V_{s h}=V_{j S h}-V_{c h} \cong\left(1.15-1.3 \cdot \frac{P_{u}}{f_{c}^{\prime} \cdot A_{g}}\right) \cdot T_{s}
$$

The term $V_{c h}$ is to be carried by the concrete compression strut while the term $V_{s h}$ will be carried by the horizontal reinforcement within the joint core.

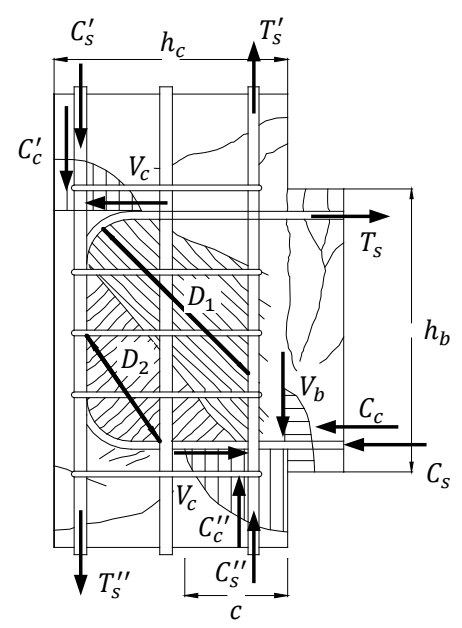

Figure 8 Exterior Joint: external actions and internal stress resultants. The compression strut is included between the two boundary lines $D_{1}$ and $D_{2}$.

Likewise, for an exterior joint, the horizontal shear force is given by:

$$
V_{j S h}=T_{s}-V_{c o l}
$$

where the value of the tension force $T_{s}$ is either $f_{s} \cdot A_{s}$ or $\lambda_{0} \cdot f_{y} \cdot A_{s}$, depending on whether an elastic beam section or the critical section of a beam plastic hinge at the face of the column is being considered. Even in this case, a diagonal strut similar to that shown in Fig. 8 will develop between the bend of the hooked top tension beam bars and the lower right-hand corner of the joint, where compression forces in both the horizontal and vertical directions are introduced. Similarly to what already introduced for the case of the interior joints, the horizontal component of the compression strut mechanism is given by:

$$
V_{c h}=C_{c}+\Delta T_{c}-V_{c o l}
$$

where: $\Delta T_{c}$ is the fraction of the steel compression force $C_{s}$ developed in the bottom beam reinforcement, introduced to the strut by means of bond over the length of bar subjected to transverse compression from the lower column.

Even in this case, based on reasonable assumptions about the likely distribution of the shear tangential stresses along the longitudinal reinforcement within the joint core, Paulay and Priestley [6] proposed the following expression to evaluate the fraction of shear force that has to be carried by the horizontal reinforcement in the joint: 


$$
V_{s h}=V_{j S h}-V_{c h} \cong \frac{\beta}{\lambda_{0}} \cdot\left(0.7-\frac{P_{u}}{f_{c}^{\prime} \cdot A_{g}}\right) \cdot T_{s}
$$

and the complement to $V_{j S h}$ will flow in the compression strut mechanism.

\subsection{Code-suggested model}

As already outlined, the Italian Code [2,5] (implicitly) distinguishes between the case of new and existing buildings. For the former [2] distinction in made between joints that are 1) fully confined or 2) not fully confined. The fully confined joints are those in which beams frame into all four sides and their width is at least three quarters of the parallel cross-sectional dimension of the column and on both pairs of opposite faces of the joint, beam cross sections cover each other for at least 3/4 of their depth. Not fully confined joints are all those not belonging to the previous category.

The horizontal shear acting on the core of a joint shall be determined taking into account the most adverse conditions under seismic actions. In absence of more accurate evaluations, shear force acting on the concrete core of the joint can be calculated, for each direction of the seismic action, by the following expressions:

$$
\begin{gathered}
V_{j b d}=\gamma_{R d} \cdot A_{s 1} \cdot f_{y d}-V_{c} \text { for exterior joints } \\
V_{j b d}=\gamma_{R d} \cdot\left(A_{s 1}+A_{s 2}\right) \cdot f_{y d}-V_{c} \text { for interior joints }
\end{gathered}
$$

where: $\gamma_{R d}=1.2$ is the factor to account for overstrength due to steel strain-hardening, $A_{s 1}$ and $A_{s 2}$ are the beam cross section top and bottom steel reinforcement, respectively; $f_{y d}$ is the steel design value of yield strength; and $V_{c}$ is the shear force in the column above the joint. Strength verification should be carried out only for joints of high ductility buildings.

Joint shear strength associated to the concrete strut crushing $V_{j R d 1}$ can be evaluated by:

$$
V_{j R d 1}=\eta \cdot f_{c d} \cdot b_{j} \cdot h_{j c} \cdot \sqrt{1-\frac{v_{d}}{\eta}}
$$

in which:

$$
\begin{gathered}
\eta=\alpha_{j} \cdot\left(1-\frac{f_{c k}}{250}\right) \text { with } f_{c k} \text { in } M P a \\
\alpha_{j}=\left\{\begin{array}{c}
0.6 \text { for interior joints } \\
0.48 \text { for exterior joints }
\end{array}\right.
\end{gathered}
$$

$f_{c k}, f_{c d}$ are concrete cylindrical strength's characteristic and design value, respectively; $v_{d}=$ $N_{S d} /\left(A_{c} \cdot f_{c d}\right)$ is the axial force in the column above the joint normalized with respect to the compressive strength of the concrete section; $h_{j c}$ is the distance between the outermost longitudinal steel bars alignments; and $b_{j}$ is the joint effective width, assumed as the minimum between 1) the larger between column and beam section width and 2) the smaller between column and beam section width, both increased by half of the column cross section depth.

Adequate confinement of the joint, both horizontal and vertical, should be provided, to limit the maximum diagonal tensile stress of concrete to the concrete design tensile strength $f_{c t d}$. In the absence of a more precise model, this requirement may be satisfied by providing horizontal hoops with a diameter of not less than $6 \mathrm{~mm}$ within the joint, such that: 


$$
\frac{A_{s h} \cdot f_{y w d}}{b_{j} \cdot h_{j w}} \geq \frac{V_{j b d}^{2}}{\left(b_{j} \cdot h_{j c}\right)^{2} \cdot\left(f_{c t d}+v_{d} \cdot f_{c d}\right)}-f_{c t d}
$$

where: $A_{s h}$ is the total area of horizontal hoops, $V_{j b d}$ is given by Eq. (24), $h_{j w}$ is the distance between the top and the bottom reinforcement of the beam, $h_{j c}$ is the distance between extreme layers of column reinforcement, $b_{j}$ is the joint effective width.

It must be stressed that some perplexity arises when Eq. (28) is analyzed in depth since it seems to be based on a contradiction. In fact, while it is purposely meant to design steel hoops in order to keep the joint concrete un-cracked, it relies on the steel yield strength $f_{y d}$ that can only be attained if the steel has actually yielded, which means that the steel bars elongation has reached $\varepsilon_{y d} \cong 20 \%$. However, such strain value can only be attained if the steel bars have undergone large elongation, across the formed cracks. Provided that further research is desirable in order to overcome such contradictions, it must also be stressed that the $\mathrm{CAM}^{\circledR}$ technique, due to the inherent pre-tension, applies a confinement without need of deformations thus resulting much more effective, in keeping the joint concrete un-cracked, than any other passive available technique, including the use of FRPs.

As an alternative, the integrity of the joint after diagonal cracking may be ensured by horizontal hoop reinforcement. To this end the following total area of horizontal hoops should be provided in the joint:

$$
\begin{gathered}
A_{s h} \cdot f_{y w d} \geq \gamma_{R d} \cdot\left(A_{s 1}+A_{s 2}\right) \cdot f_{y d} \cdot\left(1-0.8 \cdot v_{d}\right) \text { for interior joints } \\
A_{s h} \cdot f_{y w d} \geq \gamma_{R d} \cdot A_{s 2} \cdot f_{y d} \cdot\left(1-0.8 \cdot v_{d}\right) \text { for exterior joints }
\end{gathered}
$$

\subsection{Confinement-modified model}

In the present section, a first proposal is made about a possible way to evaluate the amount of reinforcement to strengthen the joints. Even though we highly support the adoption of active techniques, such as the $\mathrm{CAM}^{\circledR}$ [13], the formulation herein proposed is actually applicable also to the case of passive confinements such as the FRPs jacketing. The preference for active methods is due to the fact that confinement does not need any structure deformation to be activated but, on the contrary, it is readily available.

Conservatively, and contrarily to what suggested by Paulay and Priestley [6], we do not distinguish the amount of the overall joint shear force $V_{j b d}$ that is carried by the concrete strut $V_{c h}$ and the truss $V_{s h}$. In fact, we assume that the truss does not form due to the reason that, in order for it to be actually mobilized, horizontal reinforcement should be diffuse while, for an existing building, such reinforcement is always rather discrete, due to the complex geometrical boundary conditions. Given that, the joint verification is carried out by comparing the shear force $V_{j b d}$ with the shear strength that can be evaluated as follows:

$$
V_{j R d}=\min \left(V_{j R d 1} ; V_{j R d 2} ; V_{j R d 3}\right)
$$

in which: $V_{j R d 1}$ is the joint shear strength associated to concrete strut crushing; $V_{j R d 2}$ is the joint shear strength associated to concrete diagonal cracking and $V_{j R d 3}$ is the joint shear strength associated to the steel stirrups failure, in case they are actually present. Even though the NTC2008 considers the term associated to concrete diagonal cracking, it may not be considered since it is too conservative for a calculation carried out at the ultimate limit states, in which joints are definitely cracked. In fact, joints are supposed to be cracked since the serviceability limit state, 
through which the structure has already gone for values of $u_{c}$ much smaller than those corresponding to the ultimate state.

The joint shear force can be evaluated, as function of the control point displacement, as follows:

$$
\begin{gathered}
V_{j S d}=\frac{M_{s d}^{l}\left(u_{c}\right)}{d^{l}}+\frac{M_{s d}^{r}\left(u_{c}\right)}{d^{r}}-V_{c}\left(u_{c}\right) \text { for interior joints } \\
V_{j S d}=\frac{M_{s d}\left(u_{c}\right)}{d}-V_{c}\left(u_{c}\right) \text { for exterior joints }
\end{gathered}
$$

in which $M_{s d}^{l}\left(u_{c}\right)$ and $M_{s d}^{r}\left(u_{c}\right)$ are the bending moments applied by the two beams framing into the joint, left and rightwards respectively.

The term $V_{j R d 2}$, which is herein reported for sake of completeness, can be evaluated as follows:

$$
V_{j R d 2}=b_{j} \cdot h_{j c} \cdot \sqrt{f_{c t d} \cdot\left(f_{c t d}+v_{d} \cdot f_{c d}\right)}
$$

in which $f_{c t d}$ is concrete design tensile strength.

The term $V_{j R d 3}$ can be evaluated as follows:

$$
V_{j R d 3}=A_{s h} \cdot f_{y w d}
$$

The term $V_{j R d 1}$ can be evaluated by Eq. (25) in which $f_{c d}$ has to be substituted by the design cylindrical strength of the confined concrete $f_{c c d}$. The cylindrical strength $f_{c c}$ of the confined concrete may be evaluated by the method proposed by Braga et al. in [14]. This model allows the whole stress strain $\sigma_{z}-\varepsilon_{z}$ constitutive law of the confined concrete to be evaluated, as increment with respect to the constitutive law of the unconfined concrete, as follows:

$$
\sigma_{z}\left(\varepsilon_{z}\right)=\sigma_{z 0}\left(\varepsilon_{z}\right)+\Delta \sigma_{z}\left(\varepsilon_{z}\right)
$$

where: $\sigma_{z}$ and $\sigma_{z 0}$ are the stresses of the confined and unconfined concrete respectively and $\varepsilon_{z}$ is the related strain. The term $\Delta \sigma_{z}$ represents the stress increment related to the triaxial state. The curve $\sigma_{z 0}\left(\varepsilon_{z}\right)$ can be assumed as the classical parabola-rectangle diagram (Fig. 9) adopted by [2]. The model is based on the assumptions of 1) elastic material, 2) plain strain condition and 3) passive confinement.

Even though we are taking into consideration the case of existing RC buildings, in which case the joint does not contain any steel stirrups, the model contemplates the possibility to have complex confinement systems, with multiple horizontal hoops (Fig. 9). For each $i$-th hoop, the radial confining pressure $f_{r i}$ can be evaluated, for each value of $\varepsilon_{z}$, and assuming null bending inertia for each confining strip, be it made of either FRP or high strength steel as in the case of $\mathrm{CAM}^{\circledR}$. For a square section column the following expression is provided [14]:

$$
f_{r i}\left(\varepsilon_{z}\right)=\frac{36 \cdot E_{c} \cdot E_{m} \cdot t_{m} \cdot\left(b_{m} / S\right) \cdot v}{25 \cdot E_{c} \cdot d+24 \cdot E_{m} \cdot t_{m} \cdot\left(b_{m} / S\right) \cdot(2 \cdot v+5)} \cdot \varepsilon_{z}
$$

where: $d$ is the square cross-section dimension; $E_{c}$ and $E_{m}$ are the Young's modulus of the concrete and the confining material, respectively; $t_{m}$ and $b_{m}$ are the wrapping depth and width, respectively; $S$ is the wrapping spacing and $v$ is the concrete Poisson's ratio. Whereas, for circular section columns with external radius $R_{c e}$, the confining pressure is expressed by the following equation:

$$
f_{r i}\left(\varepsilon_{z}\right)=\frac{E_{c} \cdot E_{m} \cdot t_{m} \cdot\left(b_{m} / S\right) \cdot v}{E_{c} \cdot R_{c e}+E_{m} \cdot t_{m} \cdot\left(b_{m} / S\right) \cdot(1-v) \cdot\left(\varepsilon_{z} \cdot v+1\right)} \cdot \varepsilon_{z}
$$


and in case continuous wrapping is used, the ratio $b_{m} / S=1$.
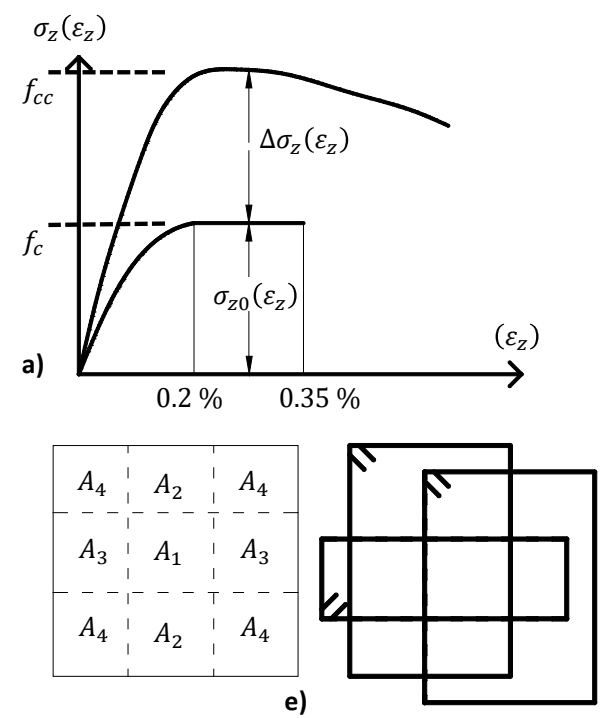

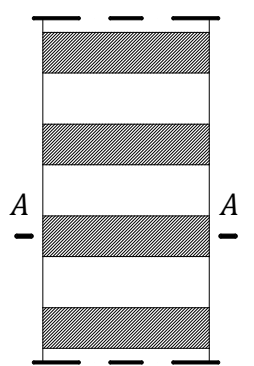

b)

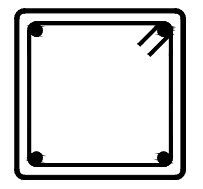

Section $A A$

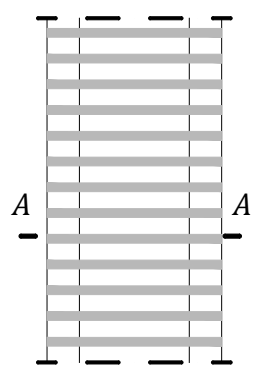

c)

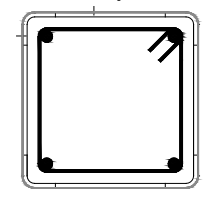

Sec. $A A$

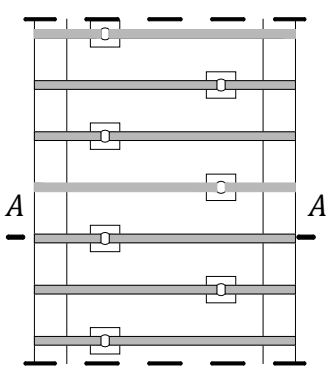

d)

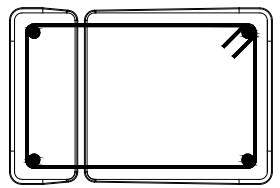

Sec. $A A$

Figure 9 Confined concrete stress-strain relationship by means of the model by Braga et al. [14]: a) increment over the unconfined concrete constitutive law; details of a square RC cross section column strengthened by either b) FRP or c) $\mathrm{CAM}^{\circledR}$ wrappings, respectively; d) rectangular cross section strengthened by $\mathrm{CAM}^{\circledR}$, and e) case of complex confinement system (very general).

In the most general case of existing internal stirrups and external wrapping, the average radial confining pressure can be evaluated as follows:

$$
f_{r m}\left(\varepsilon_{z}\right)=\frac{A_{\text {int }}}{A_{\text {ext }}} f_{r, \text { int }}+f_{r, \text { ext }}
$$

where: $f_{r, \text { int }}$ and $f_{r, \text { ext }}$ are the radial confining pressures induced by the internal stirrups and by the external wrapping, respectively; $A_{\text {int }}$ is the amount of area of the cross section within the internal stirrup and $A_{\text {ext }}$ is the area contained within the external wrapping. In the most general configuration of confinement reinforcement (Fig. 9e), the superposition method is applied to evaluate the average radial confining pressure, as follows:

$$
f_{r m}\left(\varepsilon_{z}\right)=\frac{1}{A_{e}} \cdot\left(f_{r A 1} \cdot A_{1}+2 \cdot f_{r A 2} \cdot A_{2}+2 \cdot f_{r A 3} \cdot A_{3}+4 \cdot f_{r A 4} \cdot A_{4}\right)
$$

where:

$$
f_{r A 1}=f_{r 2}+2 \cdot f_{r 1} ; f_{r A 2}=2 \cdot f_{r 1} ; f_{r A 3}=f_{r 2}+f_{r 1} ; f_{r A 4}=f_{r 1}
$$

The pressures applied by the stirrups are actually spread in the concrete along the longitudinal development of the RC element, by means of two mechanisms that are: 1) the arch effect, which develops between two adjacent stirrups, thus reducing the real confined concrete volume, and 2) the mechanism due to the bending stiffness of the longitudinal bars. As a result, the confining pressure varies along the element with maximum value at the tie level and minimum values midway between two stirrups. Therefore, the concrete is entirely subject to a lateral confining pressure lower than the mean value calculated at tie level when assuming a homogeneous distribution. Two reduction coefficients can be evaluated, separately, for each of the mechanisms above. For the concrete mechanism, the coefficient can be evaluated by the following equation: 


$$
k_{c}=\left(1-\frac{s}{4 \cdot l_{s t}}\right)^{2}
$$

where: $l_{s t}$ is the length of the stirrup. For the reductive coefficient associated to the bending stiffness of the longitudinal bars, the following expression is provided:

$$
k_{s l}=\frac{45 \cdot \xi_{l}^{3}}{45 \cdot \xi_{l}^{3}+\beta \cdot \xi_{s t}}
$$

where:

$$
\xi_{l}=\frac{\phi_{\text {long }}}{S} ; \beta=\frac{\phi_{s t}}{\phi_{\text {long }}} ; \xi_{\text {st }}=\frac{\phi_{s t}}{l}
$$

in which: $\phi_{s t}$ and $\phi_{\text {long }}$ are the diameters of the stirrup and longitudinal bar, respectively; $l$ is the element length. When the bending stiffness of the longitudinal bars becomes negligible, only the arching effect between two stirrups spreads the confining pressures along the column and it can be assumed that $k_{s l} \gg k_{c}$. However, in the most general case, the effective confining pressure to which the column (i.e. the joint panel) confined by either square or circular hoops is subject, is given by the following expression:

$$
f_{r}=k_{c} \cdot k_{s l} \cdot f_{r m}
$$

and the corresponding value of the stress increment is given by:

$$
\Delta \sigma_{z}\left(\varepsilon_{z}\right)=\Delta \sigma_{z}\left(f_{r m}\right)=-2 \cdot v \cdot f_{r m}
$$

The strength of the confined concrete is thus given by:

$$
f_{c c}=\max \left(\sigma_{z}\left(\varepsilon_{z}\right)\right)
$$

and this value has to be implemented into the Eq. (25) in order to obtain the value of $V_{j R d 1}$.

The weak points of this model are that 1) the whole constitutive law of the confined concrete has to be evaluated first, in order to obtain the relevant strength $f_{c c}$, and 2) the confinement contemplated is the passive one. Further research is, in this respect, desirable and already being carried out.

\section{OVERALL DISSIPATIVE MECHANISM}

In this context, the retrofitting design strategy may be based on the following steps, to be undertaken in sequence:

Evaluate, by means of the simple models described in previous paragraph $\S .3$, the ratio between $u_{c, y b}$ and $u_{c, y c}$;

If it happens that:

$$
u_{c, y b}<u_{c, y c}
$$

the classical overall dissipative mechanism can be adopted and the strengthening of the joints can be calculated by means of the model described in the paragraph $\S .4 .3$;

If it happens that:

$$
u_{c, y c}<u_{c, y b}
$$

the classical overall dissipative mechanism can be tentatively pursued by adopting either one of the following strategies: 1) moments redistribution or 2) repositioning of the plastic hinges;

If it happens that: 


$$
u_{c, y c} \ll u_{c, y b}
$$

an hybrid beam-column-sway mechanism should be accepted and identified by means of an energy-based approach.

Further research is already being carried out in order to formalize this procedure.

\section{CONCLUSIONS}

This work addressed the problem of the optimization of the amount of strengthening to be implemented for joints of existing RC buildings in a seismic retrofitting intervention. In fact the retrofitting design activity that has characterized the aftermath of the recent Italian earthquakes has shown that the application of the cogent Italian Building Code may lead to excessively conservative interventions that would result both difficult to realize and detrimental to the diffusion of innovative and promising strengthening techniques.

The first step to be undertaken during the design of a retrofitting intervention is the selection of a suitable overall dissipative mechanism. While a beam-sway overall dissipative mechanism can be pursued with relative ease for new buildings by means of the Capacity Design Philosophy, it may lead to unnecessarily conservative solutions for existing buildings. In fact, since these latter were most probably designed for gravity loads only, they are characterized by the Strong-Beam-Weak-Column typology. Thus, for these buildings, an alternative overall mechanism, characterized by plasticization on both beams and columns, may be selected.

A simple model was developed to help the designers figure out which overall dissipative mechanism is more suitable to pursue for the specific case study they are dealing with. Such model is based on the evaluation and comparison of the value of the displacement of the control point of the single Moment Resisting frame corresponding to the flexural yielding of beams and columns. In cases in which, due to the presence of strong beams and weak columns, these latter yield much earlier than beams, the hybrid column-beam-sway mechanism should be selected.

After reviewing the model for shear strengthening of joints implemented by the cogent Italian Building Code and the one from which it was derived, a way to improve it was suggested, in absence of a newly developed model. The beneficial effect of confinement on the concrete strut resisting mechanism may be accounted for by evaluating the confined concrete strength by means of the model by Braga et al., which has been briefly described.

Ultimately, the energy-based strategy to design the hybrid overall dissipative mechanism was described. Anyway, in this respect, further research is necessary.

This work implicitly addressed the Moment Resisting Framed typology of RC buildings, adopting simplified models. As further developments, the case of dual systems, characterized by the presence of frames and walls in parallel to each other will be also addressed. Moreover, the way in which to deal with the presence of stairs, with either cantilever steps or a climbing slab, will be also analyzed.

\section{REFERENCES}

[1] Royal Decree Law n²2291, (1939), "Norme per l'esecuzione delle opere in congolmerato cementizio semplice od armato", published on Gazzetta Ufficiale della Repubblica Italiana, $\mathrm{n}^{\circ}$ 92, 18 April 1940.

[2] NTC 2008 - DM 14 Gennaio 2008. Nuove norme tecniche per le costruzioni. Gazzetta Ufficiale della Repubblica Italiana, 4 febbraio 2008, n. 29. 
[3] UNI EN 1998-1, Eurocode 8 - Design of structures for earthquake resistance - Part 1: General rules, seismic actions and rules for buildings. CEN European Committee for Standardization.

[4] UNI EN 1992-1-1, Eurocode 2: Design of concrete structures - Part 1-1: General rules and rules for buildings. CEN European Committee for Standardization.

[5] Circolare dei Lavori Pubblici $n^{\circ} 617$. Istruzioni per l'applicazione delle nuove Norme tecniche per le Costruzioni di cui al Decreto Ministeriale 2008. Italian Ministry of Public Works, April 2009; 2009.

[6] T. Paulay, M.J.N. Priestley, Seismic design of reinforced concrete and masonry buildings, John Wiley \& Sons, Inc., 1992.

[7] H. Akiyama, Earthquake Resistant Limit-State Design for Buildings, University of Tokyo Press, 1985.

[8] C.H. Uang, V.V. Bertero, Evaluation of seismic energy in structures, Earthquake Engineering and Structural Dynamics, 19, 77-90, 1990.

[9] M.D. Symans, F.A. Charney, A.S. Whittaker, M.C. Constantinou, C.A. Kircher, M.W. Johnson, R.J. McNamara, Energy Dissipation Systems for Seismic Applications: Current Practice and Recent Developments, Journal of Structural Engineering, ASCE, January 2008 .

[10] V. Bianco, S. Granati, Exploring the possibility to carry out an expeditious non linear static assessment of an existing RC building, XV Convegno ANIDIS, Associazione Nazionale Italiana Di Ingegneria Sismica, Padova, Italy, 30 Giugno-4Luglio, 2013.

[11] V. Bianco, S. Granati, Expeditious seismic assessment of existing moment-resisting frame reinforced concrete buildings: proposal of a calculation method, Engineering Structures, 101, 715-732, 2015.

[12] R.W. Clough, J. Penzien, Dynamics of Structures, Computers and Structures, Inc., Berkley, CA, 1995.

[13] M. Dolce, R. Gigliotti, M. Laterza, D. Nigro, R. Marnetto, Il rafforzamento di pilastri in c.a. mediante il sistema CAM, $10^{\text {th }}$ International Conference L'ingegneria Sismica in Italia, Potenza-Matera, Italy, September 9-13, 2001.

[14] F. Braga, R. Gigliotti, M. Laterza, Analytical Stress-Strain Relationship for Concrete Confined by Steel Stirrups and/or FRP Jackets, Journal of Structural Engineering, ASCE, 132(9), 1402-1416, 2006. 